\title{
SELECTING VARIOUS INDUSTRIAL COMPETITORS AFFECT THE RISK LEVEL OF VIET NAM MANUFACTURING MATERIAL INDUSTRY DURING AND AFTER THE GLOBAL CRISIS 2007-2009
}

\author{
Dinh Tran Ngoc Huy \\ Banking University HCMC Viet nam - GSIM Intl University of Japan, Japan \\ E-mail: dtnhuy2010@gmail.com
}

Received August 2015; accepted November 2015

\begin{abstract}
s
Using a one factor model, this paperwork estimates the impacts of the size of firms' competitors in the manufacturing material industry on the market risk level, measured by equity and asset beta, of 99 listed companies in this category. This study identified that the risk dispersion level in this sample study could be minimized in case the competitor size doubles (measured by equity beta var of 0,293 ). Beside, the empirical research findings show us that asset beta max value increases from 1,162 to 1,445 when the size of competitor doubles. Last but not least, most of beta values are acceptable except a few exceptional cases. Ultimately, this paper illustrates calculated results that might give proper recommendations to relevant governments and institutions in re-evaluating their policies during and after the financial crisis 2007-2011.
\end{abstract}

\section{Research paper}

Keywords: Risk management, competitive firm size, market risk, asset and equity beta, manufacturing material industry

Reference to this paper should be made as follows: Huy. D. T. N. (2016). "Selecting Various Industrial Competitors Affect The Risk Level of Viet Nam Manufacturing Material Industry During and After The Global Crisis 2007-2009”, Journal of Entrepreneurship, Business and Economics, Vol. 4, No. 1, pp. 108-127.

\section{Introduction}

Together with financial system development and the economic growth, throughout many recent years, Viet Nam manufacturing material industry is considered as one of active economic sectors, which has some positive effects for the economy. Additionally, financial risk and reactions has become an issue after the global crisis 2007-2009 which has some certain impacts on the whole Vietnam economy, and specifically, the Viet Nam manufacturing 
material industry. Hence, this research paper analyzes market risk under a one factor model of these listed firms during this period.

This paper is organized as follow. The research issues and literature review will be covered in next sessions 2 and 3, for a short summary. Then, methodology and conceptual theories are introduced in session 4 and 5. Session 6 describes the data in empirical analysis. Session 7 presents empirical results and findings. Next, session 8 covers the analytical results. Then, session 9 will conclude with some policy suggestions. This paper also supports readers with references, exhibits and relevant web sources.

\section{Research Issues}

For the estimating of impacts of a one factor model: the size of competitor on beta for listed manufacturing material industry companies in Viet Nam stock exchange, research issues will be mentioned as following:

Issue 1: Whether the risk level of manufacturing material industry firms under the different changing scenarios of the size of competitor increase or decrease so much.

Issue 2: Whether the disperse distribution of beta values become large in the different changing scenarios of the size of competitor in the manufacturing material industry.

\section{Literature review}

Black (1976) proposes the leverage effect to explain the negative correlation between equity returns and return volatilities. Diamond and Dybvig (1983) said banks can also help reduce liquidity risk and therefore enable long-term investment. 
Next, Kim et al (2002) noted that the nature of competitive interaction in an industry is important in assessing the effect of corporate product strategies on shareholder value. Pagano and Mao (2007) stated that An intermediated market can therefore remain viable in the face of competition from a possibly faster, non-intermediated market as long as the specialist can generate revenue for the above services that covers his/her costs associated with asymmetric information, order processing, and inventory management. Daly and Hanh Phan (2013) investigated the competitive structure of the banking industries in five emerging Asian countries including Viet Nam and showed that the global financial crisis affected dramatically the competition of banking system in emerging Asian countries.

Last but not least, Ana and John (2013) Binomial Leverage - Volatility theorem provides a precise link between leverage and volatility.

\section{Conceptual theories}

The impact of competition or the size of competitor on the economy and business

In a specific industry such as manunfacturing material industry, there are many firms offering the similar products and services and this helps customers select a variety of qualified goods that meet their demand. Competitors could affect price and customer service policies; hence, affect revenues and profits of a typical company. The competition could drive down profits that firms can earn. Sources of competition include, but not limit to, training. Increasing training can help competition raising productivity.

\section{Methodology}


In this research, analytical research method is used, philosophical method is used and specially, scenario analysis method is used. Analytical data is from the situation of listed manufacturing material industry firms in $\mathrm{VN}$ stock exchange and applied current tax rate is $25 \%$.

Finally, we use the results to suggest policy for both these enterprises, relevant organizations and government.

\section{General Data Analysis}

The research sample has total 99 listed firms in the manufacturing material industry market with the live data from the stock exchange.

Firstly, we estimate equity and asset beta values of these firms, as well as the risk dispersion. Secondly, we change the competitor size from aprroxiamte size to doubling size and slightly smaller size to see the sensitivity of beta values. We figure out that in 3 cases, asset beta mean values are estimated at $0,371,0,392$ and 0,360 which are positively correlated with the size of competitors. Also in 3 scenarios, we find out equity beta mean values $(0,747,0,786$ and 0,728$)$ are also positively correlated with the competitive firm size. Various competitors selected definitely have certain effects on asset and equity beta values.

\section{Empirical Research Findings and Discussion}

In the below section, data used are from total 99 listed manufacturing material industry companies on VN stock exchange (HOSE and HNX mainly). In the scenario 1, current financial leverage degree is kept as in the 2011 financial statements which is used to calculate market risk (beta) whereas competitor size is kept as current, then changed from double size to slightly 
smaller size. Then, two (2) FL scenarios are changed up to $30 \%$ and down to $20 \%$, compared to the current FL degree. In short, the below table 1 shows three scenarios used for analyzing the risk level of these listed firms. Market risk (beta) under the impact of tax rate, includes: 1) equity beta; and 2) asset beta.

Table 1. Analyzing market risk under three (3) scenarios (Made by Author)

\begin{tabular}{|l|l|}
\hline & FL as current \\
\hline Competitor size as current & Scenario 1 \\
\hline Competitor size slightly smaller & Scenario 2 \\
\hline Competitor size double & Scenario 3 \\
\hline
\end{tabular}

Scenario 1: current financial leverage and competitor size kept as current In this case, all beta values of 99 listed firms on VN manufacturing material industry market as following:

Table 2. Market risk of listed companies on VN manufacturing material industry market under one factor model (case 1) (source: VN stock exchange 2012)

\begin{tabular}{|l|l|l|l|l|l|}
\hline $\begin{array}{l}\text { Orde } \\
\text { r No. }\end{array}$ & $\begin{array}{l}\text { Company } \\
\text { stock code }\end{array}$ & $\begin{array}{l}\text { Equit } \\
\text { y beta }\end{array}$ & $\begin{array}{l}\text { Asset beta (assume } \\
\text { debt beta = 0) }\end{array}$ & Note & $\begin{array}{l}\text { Financial } \\
\text { leverage (F.S } \\
\text { reports) }\end{array}$ \\
\hline 1 & COM & 0,604 & 0,473 & & $17,3 \%$ \\
\hline 2 & AAA & 0,403 & 0,186 & $\begin{array}{l}\text { VID as } \\
\text { comparable }\end{array}$ & $43,1 \%$ \\
\hline
\end{tabular}




\begin{tabular}{|c|c|c|c|c|c|}
\hline 3 & ALV & 0,890 & 0,618 & $\begin{array}{l}\text { MMC as } \\
\text { comparable }\end{array}$ & $24,5 \%$ \\
\hline 4 & AMC & 0,781 & 0,450 & $\begin{array}{l}\text { CPC as } \\
\text { comparable }\end{array}$ & $33,9 \%$ \\
\hline 5 & APP & 0,799 & 0,474 & $\begin{array}{l}\mathrm{CPC} \text { as } \\
\text { comparable }\end{array}$ & $32,5 \%$ \\
\hline 6 & BGM & 0,719 & 0,672 & $\begin{array}{l}\text { GTA as } \\
\text { comparable }\end{array}$ & $5,3 \%$ \\
\hline 7 & BKC & 1,339 & 0,928 & & $24,6 \%$ \\
\hline 8 & BMC & 1,433 & 1,036 & & $22,1 \%$ \\
\hline 9 & BMJ & $-1,712$ & $-1,377$ & & $15,7 \%$ \\
\hline 10 & BRC & 0,835 & 0,587 & $\begin{array}{l}\text { TPP as } \\
\text { comparable }\end{array}$ & $23,8 \%$ \\
\hline 11 & BVG & 0,197 & 0,053 & $\begin{array}{l}\text { COM as } \\
\text { comparable }\end{array}$ & $58,7 \%$ \\
\hline 12 & BVN & 0,531 & 0,163 & $\begin{array}{l}\text { BMC as } \\
\text { comparable }\end{array}$ & $55,5 \%$ \\
\hline 13 & CAP & 0,543 & 0,205 & $\begin{array}{l}\mathrm{CPC} \text { as } \\
\text { comparable }\end{array}$ & $49,7 \%$ \\
\hline 14 & CMI & 0,875 & 0,384 & $\begin{array}{l}\text { KKC as } \\
\text { comparable }\end{array}$ & $44,9 \%$ \\
\hline 15 & CPC & 1,211 & 0,937 & & $18,1 \%$ \\
\hline 16 & CTM & 0,350 & 0,178 & $\begin{array}{l}\text { DTT as } \\
\text { comparable }\end{array}$ & $39,4 \%$ \\
\hline 17 & $\mathrm{CZC}$ & 0,090 & 0,028 & $\begin{array}{l}\text { HVT as } \\
\text { comparable }\end{array}$ & $54,9 \%$ \\
\hline 18 & DAG & 0,435 & 0,134 & $\begin{array}{l}\text { DHC as } \\
\text { comparable }\end{array}$ & $55,4 \%$ \\
\hline 19 & DHC & 1,170 & 0,461 & & $48,5 \%$ \\
\hline 20 & $\overline{\text { DHM }}$ & 0,432 & 0,240 & $\begin{array}{l}\text { HGM as } \\
\text { comparable }\end{array}$ & $35,5 \%$ \\
\hline 21 & DLG & 0,055 & 0,014 & $\begin{array}{l}\text { SQC as } \\
\text { comparable }\end{array}$ & $59,3 \%$ \\
\hline 22 & DNS & 0,076 & 0,025 & $\begin{array}{l}\text { BVG as } \\
\text { comparable }\end{array}$ & $54,3 \%$ \\
\hline 23 & DNY & 0,063 & 0,018 & $\begin{array}{l}\text { SQC as } \\
\text { comparable }\end{array}$ & $56,4 \%$ \\
\hline 24 & DPM & 0,785 & 0,686 & & $10,0 \%$ \\
\hline 25 & DPR & 1,043 & 0,808 & & $18,0 \%$ \\
\hline 26 & DTL & 0,027 & 0,011 & $\begin{array}{l}\text { DLG as } \\
\text { comparable }\end{array}$ & $47,2 \%$ \\
\hline 27 & DTT & 0,605 & 0,517 & & $11,6 \%$ \\
\hline 28 & GER & 0,746 & 0,419 & $\begin{array}{l}\text { MMC as } \\
\text { comparable }\end{array}$ & $35,1 \%$ \\
\hline 29 & GTA & 0,757 & 0,569 & & $19,9 \%$ \\
\hline
\end{tabular}


Huy. D. T. N. 2016. Selecting Various Industrial Competitors Affect The Risk Level of Viet Nam Manufacturing Material Industry During and After The Global Crisis 2007-2009

\begin{tabular}{|c|c|c|c|c|c|}
\hline 30 & HAI & 0,823 & 0,456 & & $35,7 \%$ \\
\hline 31 & HAP & 1,280 & 1,018 & & $16,4 \%$ \\
\hline 32 & HGM & 0,691 & 0,535 & & $18,0 \%$ \\
\hline 33 & HLA & 1,833 & 0,339 & & $65,2 \%$ \\
\hline 34 & HLC & 0,397 & 0,045 & & $71,0 \%$ \\
\hline 35 & HMC & 1,227 & 0,348 & & 5 \\
\hline 36 & HPG & 0,969 & 0,424 & & $45,0 \%$ \\
\hline 37 & HPP & 0,627 & 0,268 & $\begin{array}{l}\text { KMT as } \\
\text { comparable }\end{array}$ & $45,9 \%$ \\
\hline 38 & HRC & 0,971 & 0,750 & & $18,2 \%$ \\
\hline 39 & HSG & 1,821 & 0,587 & & $54,2 \%$ \\
\hline 40 & HSI & 0,748 & 0,154 & & $63,6 \%$ \\
\hline 41 & HVC & 0,314 & 0,083 & $\begin{array}{l}\text { HRC as } \\
\text { comparable }\end{array}$ & $58,9 \%$ \\
\hline 42 & HVT & 0,238 & 0,105 & & $44,6 \%$ \\
\hline 43 & KHB & 0,550 & 0,486 & $\begin{array}{l}\text { DTT as } \\
\text { comparable }\end{array}$ & $9,3 \%$ \\
\hline 44 & KKC & 1,717 & 0,860 & & $39,9 \%$ \\
\hline 45 & KMT & 1,259 & 0,386 & & $55,4 \%$ \\
\hline 46 & KSA & 0,859 & 0,530 & $\begin{array}{l}\text { KMT as } \\
\text { comparable }\end{array}$ & $30,6 \%$ \\
\hline 47 & KSB & 1,103 & 0,705 & & $28,9 \%$ \\
\hline 48 & $\mathrm{KSH}$ & 1,766 & 1,162 & & $27,4 \%$ \\
\hline 49 & KSS & 2,089 & 1,049 & & $39,8 \%$ \\
\hline 50 & KTB & 0,485 & 0,366 & $\begin{array}{l}\text { COM as } \\
\text { comparable }\end{array}$ & $19,7 \%$ \\
\hline 51 & LAS & 0,478 & 0,185 & $\begin{array}{l}\text { DPR as } \\
\text { comparable }\end{array}$ & $49,0 \%$ \\
\hline 52 & LCM & 0,542 & 0,531 & $\begin{array}{l}\text { KHB as } \\
\text { comparable }\end{array}$ & $1,6 \%$ \\
\hline 53 & MAX & 0,066 & 0,044 & $\begin{array}{l}\mathrm{CZC} \text { as } \\
\text { comparable }\end{array}$ & $26,7 \%$ \\
\hline 54 & MDC & 0,546 & 0,126 & & $61,6 \%$ \\
\hline 55 & MDF & 0,067 & 0,057 & $\begin{array}{l}\text { DNS as } \\
\text { comparable }\end{array}$ & $12,2 \%$ \\
\hline 56 & MHL & 0,482 & 0,252 & & $38,1 \%$ \\
\hline 57 & MIC & 1,417 & 0,902 & & $29,1 \%$ \\
\hline 58 & MIH & 0,068 & 0,016 & $\begin{array}{l}\text { HVT as } \\
\text { comparable }\end{array}$ & $61,6 \%$ \\
\hline 59 & MIM & 0,425 & 0,196 & $\begin{array}{l}\text { APP as } \\
\text { comparable }\end{array}$ & $43,2 \%$ \\
\hline 60 & MMC & 1,183 & 0,990 & & $13,1 \%$ \\
\hline 61 & NBC & 1,129 & 0,273 & & $60,7 \%$ \\
\hline
\end{tabular}




\begin{tabular}{|c|c|c|c|c|c|}
\hline 62 & NKG & 0,007 & 0,002 & $\begin{array}{l}\text { DTL as } \\
\text { comparable }\end{array}$ & $62,8 \%$ \\
\hline 63 & NSP & 0,811 & 0,719 & $\begin{array}{l}\text { ALV as } \\
\text { comparable }\end{array}$ & $9,1 \%$ \\
\hline 64 & NVC & 0,353 & 0,050 & & $68,6 \%$ \\
\hline 65 & $\begin{array}{l}\text { PHR } \\
\end{array}$ & 0,471 & 0,268 & & $34,5 \%$ \\
\hline 66 & $\begin{array}{l}\text { PHT } \\
\end{array}$ & 0,912 & 0,477 & & $38,2 \%$ \\
\hline 67 & PLC & 1,338 & 0,448 & & $53,2 \%$ \\
\hline 68 & POM & 0,111 & 0,038 & $\begin{array}{l}\text { TIS as } \\
\text { comparable }\end{array}$ & $52,4 \%$ \\
\hline 69 & PTK & 1,368 & 0,986 & $\begin{array}{l}\text { KSH as } \\
\text { comparable }\end{array}$ & $22,3 \%$ \\
\hline 70 & RDP & 0,827 & 0,303 & & $50,7 \%$ \\
\hline 71 & SHA & 0,810 & 0,314 & $\begin{array}{l}\text { KSH as } \\
\text { comparable }\end{array}$ & $48,9 \%$ \\
\hline 72 & SHI & 1,550 & 0,476 & & $55,4 \%$ \\
\hline 73 & SMC & 1,142 & 0,266 & & $61,3 \%$ \\
\hline 74 & SPC & 0,062 & 0,015 & $\begin{array}{l}\text { VCA as } \\
\text { comparable }\end{array}$ & $61,0 \%$ \\
\hline 75 & SQC & 0,174 & 0,148 & & $11,9 \%$ \\
\hline 76 & SSM & 1,402 & 0,710 & & $39,5 \%$ \\
\hline 77 & TC6 & 0,678 & 0,127 & & $65,0 \%$ \\
\hline 78 & TCS & 0,900 & 0,152 & & $66,5 \%$ \\
\hline 79 & TDN & 0,587 & 0,127 & & $62,7 \%$ \\
\hline 80 & TDS & 0,398 & 0,146 & $\begin{array}{l}\text { PHT as } \\
\text { comparable }\end{array}$ & $50,6 \%$ \\
\hline 81 & THT & 0,927 & 0,294 & & $54,6 \%$ \\
\hline 82 & TIS & 0,268 & 0,075 & $\begin{array}{l}\text { DPM as } \\
\text { comparable }\end{array}$ & $57,6 \%$ \\
\hline 83 & TLH & 0,320 & 0,151 & $\begin{array}{l}\text { TDN as } \\
\text { comparable }\end{array}$ & $42,2 \%$ \\
\hline 84 & TNB & 0,072 & 0,054 & $\begin{array}{l}\text { CZC as } \\
\text { comparable }\end{array}$ & $19,9 \%$ \\
\hline 85 & TNC & 0,949 & 0,846 & & $8,7 \%$ \\
\hline 86 & TNT & 1,085 & 0,781 & $\begin{array}{l}\text { SSM as } \\
\text { comparable }\end{array}$ & $22,4 \%$ \\
\hline 87 & TPC & 1,062 & 0,531 & & $40,0 \%$ \\
\hline 88 & TPP & 1,100 & 0,321 & & $56,7 \%$ \\
\hline 89 & TRC & 1,185 & 0,917 & & $18,1 \%$ \\
\hline 90 & TSC & 0,928 & 0,204 & & $62,4 \%$ \\
\hline 91 & TTF & 1,576 & 0,392 & & $60,1 \%$ \\
\hline 92 & TVD & 0,235 & 0,037 & $\begin{array}{l}\text { TRC as } \\
\text { comparable }\end{array}$ & $67,5 \%$ \\
\hline
\end{tabular}


Huy. D. T. N. 2016. Selecting Various Industrial Competitors Affect The Risk Level of Viet Nam Manufacturing Material Industry During and After The Global Crisis 2007-2009

\begin{tabular}{|l|l|l|l|l|l|}
\hline 93 & VCA & 0,212 & 0,044 & $\begin{array}{l}\text { RDP as } \\
\text { comparable }\end{array}$ & $63,5 \%$ \\
\hline 94 & VDT & 0,665 & 0,326 & $\begin{array}{l}\text { MMC as } \\
\text { comparable }\end{array}$ & $40,8 \%$ \\
\hline 95 & VFG & 0,350 & 0,181 & & $38,6 \%$ \\
\hline 96 & VGS & 1,907 & 0,820 & & $45,6 \%$ \\
\hline 97 & VID & 0,757 & 0,272 & & $51,2 \%$ \\
\hline 98 & VIS & 1,289 & 0,500 & & $49,0 \%$ \\
\hline 99 & VKP & 0,877 & 0,131 & & $68,0 \%$ \\
\hline & & & & Average & $40,8 \%$ \\
\hline
\end{tabular}

Scenario 2: competitor size double

All beta values of total 99 listed firms on VN manufacturing material industry market as below:

Table 3. Market risks of listed manufacturing material industry firms under one factor model (case 2) (source: VN stock exchange 2012)

\begin{tabular}{|c|c|c|c|c|c|}
\hline $\begin{array}{l}\text { Orde } \\
\text { r No. }\end{array}$ & $\begin{array}{l}\text { Company } \\
\text { stock code }\end{array}$ & $\begin{array}{l}\text { Equit } \\
\text { y beta }\end{array}$ & $\begin{array}{l}\text { Asset beta (assume } \\
\text { debt beta }=0 \text { ) }\end{array}$ & Note & $\begin{array}{ll}\begin{array}{l}\text { Financial } \\
\text { leverage } \\
\text { reports) }\end{array} \quad \text { (F.S } \\
\end{array}$ \\
\hline 1 & $\mathrm{COM}$ & 0,604 & 0,473 & & $17,3 \%$ \\
\hline 2 & AAA & 0,403 & 0,186 & $\begin{array}{l}\text { VID as } \\
\text { comparable }\end{array}$ & $43,1 \%$ \\
\hline 3 & ALV & 0,890 & 0,618 & $\begin{array}{l}\text { MMC as } \\
\text { comparable }\end{array}$ & $24,5 \%$ \\
\hline 4 & AMC & 0,781 & 0,450 & $\begin{array}{l}\text { CPC as } \\
\text { comparable }\end{array}$ & $33,9 \%$ \\
\hline 5 & APP & 0,799 & 0,474 & $\begin{array}{l}\text { CPC as } \\
\text { comparable }\end{array}$ & $32,5 \%$ \\
\hline 6 & BGM & 0,719 & 0,672 & $\begin{array}{l}\text { GTA as } \\
\text { comparable }\end{array}$ & $5,3 \%$ \\
\hline 7 & $\mathrm{BKC}$ & 1,339 & 0,928 & & $24,6 \%$ \\
\hline 8 & $\mathrm{BMC}$ & 1,433 & 1,036 & & $22,1 \%$ \\
\hline 9 & BMJ & $-1,712$ & $-1,377$ & & $15,7 \%$ \\
\hline 10 & BRC & 0,835 & 0,587 & $\begin{array}{l}\text { TPP as } \\
\text { comparable }\end{array}$ & $23,8 \%$ \\
\hline 11 & $\overline{B V G}$ & 0,197 & 0,053 & $\begin{array}{l}\text { COM as } \\
\text { comparable }\end{array}$ & $58,7 \%$ \\
\hline 12 & BVN & 0,531 & 0,163 & $\begin{array}{l}\text { BMC as } \\
\text { comparable }\end{array}$ & $55,5 \%$ \\
\hline 13 & CAP & 0,543 & 0,205 & CPC & $49,7 \%$ \\
\hline
\end{tabular}




\begin{tabular}{|c|c|c|c|c|c|}
\hline & & & & comparable & \\
\hline 14 & CMI & 0,875 & 0,384 & $\begin{array}{l}\text { KKC as } \\
\text { comparable }\end{array}$ & $44,9 \%$ \\
\hline 15 & CPC & 1,211 & 0,937 & & $18,1 \%$ \\
\hline 16 & CTM & 0,350 & 0,178 & $\begin{array}{l}\text { DTT as } \\
\text { comparable }\end{array}$ & $39,4 \%$ \\
\hline 17 & $\mathrm{CZC}$ & 0,090 & 0,028 & $\begin{array}{l}\text { HVT as } \\
\text { comparable }\end{array}$ & $54,9 \%$ \\
\hline 18 & DAG & 0,435 & 0,134 & $\begin{array}{l}\text { DHC as } \\
\text { comparable }\end{array}$ & $55,4 \%$ \\
\hline 19 & DHC & 1,170 & 0,461 & & $48,5 \%$ \\
\hline 20 & DHM & 0,432 & 0,240 & $\begin{array}{l}\text { HGM as } \\
\text { comparable }\end{array}$ & $35,5 \%$ \\
\hline 21 & DLG & 0,055 & 0,014 & $\begin{array}{l}\text { SQC as } \\
\text { comparable }\end{array}$ & $59,3 \%$ \\
\hline 22 & DNS & 0,076 & 0,025 & $\begin{array}{l}\text { BVG as } \\
\text { comparable }\end{array}$ & $54,3 \%$ \\
\hline 23 & DNY & 0,063 & 0,018 & $\begin{array}{l}\text { SQC as } \\
\text { comparable }\end{array}$ & $56,4 \%$ \\
\hline 24 & DPM & 0,785 & 0,686 & & $10,0 \%$ \\
\hline 25 & DPR & 1,043 & 0,808 & & $18,0 \%$ \\
\hline 26 & DTL & 0,027 & 0,011 & $\begin{array}{l}\text { DLG as } \\
\text { comparable }\end{array}$ & $47,2 \%$ \\
\hline 27 & DTT & 0,605 & 0,517 & & $11,6 \%$ \\
\hline 28 & GER & 0,746 & 0,419 & $\begin{array}{l}\text { MMC as } \\
\text { comparable }\end{array}$ & $35,1 \%$ \\
\hline 29 & GTA & 0,757 & 0,569 & & $19,9 \%$ \\
\hline 30 & HAI & 0,823 & 0,456 & & $35,7 \%$ \\
\hline 31 & HAP & 1,280 & 1,018 & & $16,4 \%$ \\
\hline 32 & HGM & 0,691 & 0,535 & & $18,0 \%$ \\
\hline 33 & HLA & 1,833 & 0,339 & & $65,2 \%$ \\
\hline 34 & HLC & 0,397 & 0,045 & & $71,0 \%$ \\
\hline 35 & HMC & 1,227 & 0,348 & & $57,3 \%$ \\
\hline 36 & HPG & 0,969 & 0,424 & & $45,0 \%$ \\
\hline 37 & HPP & 0,627 & 0,268 & $\begin{array}{l}\text { KMT as } \\
\text { comparable }\end{array}$ & $45,9 \%$ \\
\hline 38 & HRC & 0,971 & 0,750 & & $18,2 \%$ \\
\hline 39 & HSG & 1,821 & 0,587 & & $54,2 \%$ \\
\hline 40 & HSI & 0,748 & 0,154 & & $63,6 \%$ \\
\hline 41 & HVC & 0,314 & 0,083 & $\begin{array}{l}\text { HRC as } \\
\text { comparable }\end{array}$ & $58,9 \%$ \\
\hline 42 & HVT & 0,238 & 0,105 & & $44,6 \%$ \\
\hline 43 & KHB & 0,550 & 0,486 & $\begin{array}{l}\text { DTT as } \\
\text { comparable }\end{array}$ & $9,3 \%$ \\
\hline
\end{tabular}


Huy. D. T. N. 2016. Selecting Various Industrial Competitors Affect The Risk Level of Viet Nam Manufacturing Material Industry During and After The Global Crisis 2007-2009

\begin{tabular}{|c|c|c|c|c|c|}
\hline 44 & $\mathrm{KKC}$ & 1,717 & 0,860 & & $39,9 \%$ \\
\hline 45 & KMT & 1,259 & 0,386 & & $55,4 \%$ \\
\hline 46 & $\mathrm{KSA}$ & 0,859 & 0,530 & $\begin{array}{l}\text { KMT as } \\
\text { comparable }\end{array}$ & $30,6 \%$ \\
\hline 47 & KSB & 1,103 & 0,705 & & $28,9 \%$ \\
\hline 48 & $\mathrm{KSH}$ & 1,766 & 1,162 & & $27,4 \%$ \\
\hline 49 & KSS & 2,089 & 1,049 & & $39,8 \%$ \\
\hline 50 & KTB & 0,485 & 0,366 & $\begin{array}{l}\text { COM as } \\
\text { comparable }\end{array}$ & $19,7 \%$ \\
\hline 51 & LAS & 0,478 & 0,185 & $\begin{array}{l}\text { DPR as } \\
\text { comparable }\end{array}$ & $49,0 \%$ \\
\hline 52 & LCM & 0,542 & 0,531 & $\begin{array}{l}\text { KHB as } \\
\text { comparable }\end{array}$ & $1,6 \%$ \\
\hline 53 & MAX & 0,066 & 0,044 & $\begin{array}{l}\text { CZC as } \\
\text { comparable }\end{array}$ & $26,7 \%$ \\
\hline 54 & $\mathrm{MDC}$ & 0,546 & 0,126 & & $61,6 \%$ \\
\hline 55 & MDF & 0,067 & 0,057 & $\begin{array}{l}\text { DNS as } \\
\text { comparable }\end{array}$ & $12,2 \%$ \\
\hline 56 & MHL & 0,482 & 0,252 & & $38,1 \%$ \\
\hline 57 & $\mathrm{MIC}$ & 1,417 & 0,902 & & $29,1 \%$ \\
\hline 58 & $\mathrm{MIH}$ & 0,068 & 0,016 & $\begin{array}{l}\text { HVT as } \\
\text { comparable }\end{array}$ & $61,6 \%$ \\
\hline 59 & MIM & 0,425 & 0,196 & $\begin{array}{l}\text { APP as } \\
\text { comparable }\end{array}$ & $43,2 \%$ \\
\hline 60 & $\mathrm{MMC}$ & 1,183 & 0,990 & & $13,1 \%$ \\
\hline 61 & $\mathrm{NBC}$ & 1,129 & 0,273 & & $60,7 \%$ \\
\hline 62 & $\mathrm{NKG}$ & 0,007 & 0,002 & $\begin{array}{l}\text { DTL as } \\
\text { comparable }\end{array}$ & $62,8 \%$ \\
\hline 63 & NSP & 0,811 & 0,719 & $\begin{array}{l}\text { ALV as } \\
\text { comparable }\end{array}$ & $9,1 \%$ \\
\hline 64 & NVC & 0,353 & 0,050 & & $68,6 \%$ \\
\hline 65 & PHR & 0,471 & 0,268 & & $34,5 \%$ \\
\hline 66 & PHT & 0,912 & 0,477 & & $38,2 \%$ \\
\hline 67 & PLC & 1,338 & 0,448 & & $53,2 \%$ \\
\hline 68 & POM & 0,111 & 0,038 & $\begin{array}{l}\text { TIS as } \\
\text { comparable }\end{array}$ & $52,4 \%$ \\
\hline 69 & PTK & 1,368 & 0,986 & $\begin{array}{l}\mathrm{KSH} \text { as } \\
\text { comparable }\end{array}$ & $22,3 \%$ \\
\hline 70 & RDP & 0,827 & 0,303 & & $50,7 \%$ \\
\hline 71 & SHA & 0,810 & 0,314 & $\begin{array}{l}\text { KSH as } \\
\text { comparable }\end{array}$ & $48,9 \%$ \\
\hline 72 & SHI & 1,550 & 0,476 & & $55,4 \%$ \\
\hline 73 & SMC & 1,142 & 0,266 & & $61,3 \%$ \\
\hline 74 & SPC & 0,062 & 0,015 & VCA & $61,0 \%$ \\
\hline
\end{tabular}




\begin{tabular}{|c|c|c|c|c|c|}
\hline & & & & comparable & \\
\hline 75 & SQC & 0,174 & 0,148 & & $11,9 \%$ \\
\hline 76 & SSM & 1,402 & 0,710 & & $39,5 \%$ \\
\hline 77 & TC6 & 0,678 & 0,127 & & $65,0 \%$ \\
\hline 78 & TCS & 0,900 & 0,152 & & $66,5 \%$ \\
\hline 79 & TDN & 0,587 & 0,127 & & $62,7 \%$ \\
\hline 80 & TDS & 0,398 & 0,146 & $\begin{array}{l}\text { PHT as } \\
\text { comparable }\end{array}$ & $50,6 \%$ \\
\hline 81 & THT & 0,927 & 0,294 & & $54,6 \%$ \\
\hline 82 & TIS & 0,268 & 0,075 & $\begin{array}{l}\text { DPM as } \\
\text { comparable }\end{array}$ & $57,6 \%$ \\
\hline 83 & TLH & 0,320 & 0,151 & $\begin{array}{l}\text { TDN as } \\
\text { comparable }\end{array}$ & $42,2 \%$ \\
\hline 84 & TNB & 0,072 & 0,054 & $\begin{array}{l}\text { CZC as } \\
\text { comparable }\end{array}$ & $19,9 \%$ \\
\hline 85 & TNC & 0,949 & 0,846 & & $8,7 \%$ \\
\hline 86 & TNT & 1,085 & 0,781 & $\begin{array}{l}\text { SSM as } \\
\text { comparable }\end{array}$ & $22,4 \%$ \\
\hline 87 & TPC & 1,062 & 0,531 & & $40,0 \%$ \\
\hline 88 & TPP & 1,100 & 0,321 & & $56,7 \%$ \\
\hline 89 & TRC & 1,185 & 0,917 & & $18,1 \%$ \\
\hline 90 & TSC & 0,928 & 0,204 & & $62,4 \%$ \\
\hline 91 & TTF & 1,576 & 0,392 & & $60,1 \%$ \\
\hline 92 & TVD & 0,235 & 0,037 & $\begin{array}{l}\text { TRC as } \\
\text { comparable }\end{array}$ & $67,5 \%$ \\
\hline 93 & VCA & 0,212 & 0,044 & $\begin{array}{l}\text { RDP as } \\
\text { comparable }\end{array}$ & $63,5 \%$ \\
\hline 94 & VDT & 0,665 & 0,326 & $\begin{array}{l}\text { MMC as } \\
\text { comparable }\end{array}$ & $40,8 \%$ \\
\hline 95 & VFG & 0,350 & 0,181 & & $38,6 \%$ \\
\hline 96 & VGS & 1,907 & 0,820 & & $45,6 \%$ \\
\hline 97 & VID & 0,757 & 0,272 & & $51,2 \%$ \\
\hline 98 & VIS & 1,289 & 0,500 & & $49,0 \%$ \\
\hline \multirow[t]{2}{*}{99} & VKP & 0,877 & 0,131 & & $68,0 \%$ \\
\hline & & & & Average & $40,8 \%$ \\
\hline
\end{tabular}

\section{Scenario 3: Competitor size slightly smaller}

All beta values of total 99 listed firms on the manufacturing material industry market in $\mathrm{VN}$ as following: 
Huy. D. T. N. 2016. Selecting Various Industrial Competitors Affect The Risk Level of Viet Nam Manufacturing Material Industry During and After The Global Crisis 2007-2009

Table 4. Market risk of listed manufacturing material industry firms under one factor model (case 3) (source: VN stock exchange 2012)

\begin{tabular}{|c|c|c|c|c|}
\hline $\begin{array}{l}\text { Order } \\
\text { No. }\end{array}$ & $\begin{array}{l}\text { Company stock } \\
\text { code }\end{array}$ & $\begin{array}{l}\text { Equity } \\
\text { beta }\end{array}$ & $\begin{array}{l}\text { Asset beta (assume debt } \\
\text { beta }=0 \text { ) }\end{array}$ & Note \\
\hline 1 & $\mathrm{COM}$ & 0,604 & 0,473 & \\
\hline 2 & $\overline{\mathrm{AAA}}$ & 0,752 & 0,346 & $\begin{array}{ll}\text { HAP } & \text { as } \\
\text { comparable }\end{array}$ \\
\hline 3 & ALV & 0,863 & 0,599 & $\begin{array}{ll}\text { HTP } & \text { as } \\
\text { comparable }\end{array}$ \\
\hline 4 & $\mathrm{AMC}$ & 0,822 & 0,474 & $\begin{array}{ll}\text { MMC } & \text { as } \\
\text { comparable }\end{array}$ \\
\hline 5 & APP & $-1,213$ & $-0,720$ & $\begin{array}{ll}\text { BMJ } & \text { as } \\
\text { comparable }\end{array}$ \\
\hline 6 & BGM & 0,322 & 0,300 & $\begin{array}{l}\text { CZC } \\
\text { comparable }\end{array}$ \\
\hline 7 & $\mathrm{BKC}$ & 1,339 & 0,928 & \\
\hline 8 & BMC & 1,433 & 1,036 & \\
\hline 9 & BMJ & $-1,712$ & $-1,377$ & \\
\hline 10 & BRC & 0,774 & 0,544 & $\begin{array}{ll}\text { CMI } & \text { as } \\
\text { comparable } & \\
\end{array}$ \\
\hline 11 & BVG & 0,228 & 0,061 & $\begin{array}{ll}\text { COM } & \text { as } \\
\text { comparable }\end{array}$ \\
\hline 12 & BVN & 0,136 & 0,042 & $\begin{array}{ll}\text { BGM } & \text { as } \\
\text { comparable }\end{array}$ \\
\hline 13 & CAP & 0,610 & 0,231 & $\begin{array}{ll}\text { CPC } & \text { as } \\
\text { comparable }\end{array}$ \\
\hline 14 & CMI & 0,970 & 0,425 & $\begin{array}{ll}\text { KKC } & \text { as } \\
\text { comparable }\end{array}$ \\
\hline 15 & CPC & 1,211 & 0,937 & \\
\hline 16 & CTM & $-0,767$ & $-0,390$ & $\begin{array}{ll}\text { APP } & \text { as } \\
\text { comparable }\end{array}$ \\
\hline 17 & $\mathrm{CZC}$ & 0,335 & 0,105 & $\begin{array}{ll}\text { BRC } & \text { as } \\
\text { comparable }\end{array}$ \\
\hline 18 & DAG & 0,498 & 0,153 & $\begin{array}{l}\text { DHC } \\
\text { comparable }\end{array}$ \\
\hline 19 & DHC & 1,170 & 0,461 & \\
\hline 20 & DHM & 0,969 & 0,539 & $\begin{array}{ll}\text { BMC } & \text { as } \\
\text { comparable }\end{array}$ \\
\hline 21 & $\overline{\text { DLG }}$ & 0,032 & 0,008 & $\begin{array}{ll}\text { DTL } & \text { as } \\
\text { comparable }\end{array}$ \\
\hline 22 & DNS & 0,100 & 0,032 & $\begin{array}{ll}\text { BVG } & \text { as } \\
\text { comparable }\end{array}$ \\
\hline 23 & DNY & 0,163 & 0,048 & $\begin{array}{ll}\text { HLC } & \text { as } \\
\text { comparable }\end{array}$ \\
\hline
\end{tabular}




\begin{tabular}{|c|c|c|c|c|}
\hline 24 & DPM & 0,785 & 0,686 & \\
\hline 25 & DPR & 1,043 & 0,808 & \\
\hline 26 & DTL & 0,088 & 0,036 & $\begin{array}{l}\text { DNY } \\
\text { comparable }\end{array}$ \\
\hline 27 & DTT & 0,605 & 0,517 & \\
\hline 28 & GER & 0,336 & 0,188 & $\begin{array}{l}\text { VDT } \\
\text { comparable }\end{array}$ \\
\hline 29 & GTA & 0,757 & 0,569 & \\
\hline 30 & HAI & 0,823 & 0,456 & \\
\hline 31 & HAP & 1,280 & 1,018 & \\
\hline 32 & HGM & 0,691 & 0,535 & \\
\hline 33 & HLA & 1,833 & 0,339 & \\
\hline 34 & HLC & 0,397 & 0,045 & \\
\hline 35 & HMC & 1,227 & 0,348 & \\
\hline 36 & HPG & 0,969 & 0,424 & \\
\hline 37 & HPP & 0,578 & 0,247 & $\begin{array}{l}\text { KSA } \\
\text { comparable }\end{array}$ \\
\hline 38 & HRC & 0,971 & 0,750 & \\
\hline 39 & HSG & 1,821 & 0,587 & \\
\hline 40 & HSI & 0,748 & 0,154 & \\
\hline 41 & HVC & 0,186 & 0,049 & $\begin{array}{l}\text { DAG } \\
\text { comparable }\end{array}$ \\
\hline 42 & HVT & 0,238 & 0,105 & \\
\hline 43 & KHB & 0,262 & 0,231 & $\begin{array}{l}\text { MIM a } \\
\text { comparable }\end{array}$ \\
\hline 44 & KKC & 1,717 & 0,860 & \\
\hline 45 & KMT & 1,259 & 0,386 & \\
\hline 46 & KSA & 1,044 & 0,644 & $\begin{array}{l}\text { BMC } \\
\text { comparable }\end{array}$ \\
\hline 47 & KSB & 1,103 & 0,705 & \\
\hline 48 & KSH & 1,766 & 1,162 & \\
\hline 49 & KSS & 2,089 & 1,049 & \\
\hline 50 & KTB & 1,052 & 0,793 & $\begin{array}{l}\text { KMT } \\
\text { comparable }\end{array}$ \\
\hline 51 & LAS & 0,017 & 0,006 & $\begin{array}{l}\text { DLG } \\
\text { comparable }\end{array}$ \\
\hline 52 & LCM & 0,259 & 0,254 & $\begin{array}{l}\text { KHB } \\
\text { comparable }\end{array}$ \\
\hline 53 & MAX & 0,729 & 0,486 & $\begin{array}{l}\text { TNB } \\
\text { comparable }\end{array}$ \\
\hline 54 & MDC & 0,546 & 0,126 & \\
\hline 55 & MDF & 0,950 & 0,804 & $\begin{array}{l}\text { KTB } \\
\text { comparable }\end{array}$ \\
\hline
\end{tabular}


Huy. D. T. N. 2016. Selecting Various Industrial Competitors Affect The Risk Level of Viet Nam Manufacturing Material Industry During and After The Global Crisis 2007-2009

\begin{tabular}{|c|c|c|c|c|}
\hline 56 & MHL & 0,482 & 0,252 & \\
\hline 57 & MIC & 1,417 & 0,902 & \\
\hline 58 & MIH & 0,243 & 0,056 & $\begin{array}{l}\text { MAX } \\
\text { comparable }\end{array}$ \\
\hline 59 & MIM & 0,283 & 0,130 & $\begin{array}{l}\text { MHL a } \\
\text { comparable }\end{array}$ \\
\hline 60 & MMC & 1,183 & 0,990 & \\
\hline 61 & NBC & 1,129 & 0,273 & \\
\hline 62 & NKG & 0,027 & 0,006 & $\begin{array}{l}\text { DTL } \\
\text { comparable }\end{array}$ \\
\hline 63 & NSP & 0,801 & 0,710 & $\begin{array}{l}\text { ALV } \\
\text { comparable }\end{array}$ \\
\hline 64 & NVC & 0,353 & 0,050 & \\
\hline 65 & PHR & 0,471 & 0,268 & \\
\hline 66 & PHT & 0,912 & 0,477 & \\
\hline 67 & PLC & 1,338 & 0,448 & \\
\hline 68 & $\mathrm{POM}$ & 0,851 & 0,293 & $\begin{array}{l}\text { HSG } \\
\text { comparable }\end{array}$ \\
\hline 69 & PTK & 0,101 & 0,073 & $\begin{array}{l}\text { SHA } \\
\text { comparable }\end{array}$ \\
\hline 70 & RDP & 0,827 & 0,303 & \\
\hline 71 & SHA & 0,125 & 0,048 & $\begin{array}{l}\text { MIH } \\
\text { comparable }\end{array}$ \\
\hline 72 & SHI & 1,550 & 0,476 & \\
\hline 73 & SMC & 1,142 & 0,266 & \\
\hline 74 & SPC & 0,325 & 0,077 & $\begin{array}{l}\text { MDF } \\
\text { comparable }\end{array}$ \\
\hline 75 & SQC & 0,174 & 0,148 & \\
\hline 76 & SSM & 1,402 & 0,710 & \\
\hline 77 & TC6 & 0,678 & 0,127 & \\
\hline 78 & TCS & 0,900 & 0,152 & \\
\hline 79 & TDN & 0,587 & 0,127 & \\
\hline 80 & TDS & 0,160 & 0,059 & $\begin{array}{ll}\text { SPC } & \mathrm{a} \\
\text { comparable }\end{array}$ \\
\hline 81 & THT & 0,927 & 0,294 & \\
\hline 82 & TIS & 0,717 & 0,201 & $\begin{array}{l}\text { HSG } \\
\text { comparable }\end{array}$ \\
\hline 83 & TLH & 0,406 & 0,192 & $\begin{array}{l}\text { TC6 a } \\
\text { comparable }\end{array}$ \\
\hline 84 & TNB & 0,948 & 0,713 & $\begin{array}{l}\text { TNT } \\
\text { comparable }\end{array}$ \\
\hline 85 & TNC & 0,949 & 0,846 & \\
\hline 86 & TNT & 1,137 & 0,818 & $\begin{array}{l}\text { SSM } \\
\text { comparable }\end{array}$ \\
\hline
\end{tabular}




\begin{tabular}{|c|c|c|c|c|}
\hline 87 & TPC & 1,062 & 0,531 & \\
\hline 88 & TPP & 1,100 & 0,321 & \\
\hline 89 & TRC & 1,185 & 0,917 & \\
\hline 90 & TSC & 0,928 & 0,204 & \\
\hline 91 & TTF & 1,576 & 0,392 & \\
\hline 92 & TVD & 0,096 & 0,015 & $\begin{array}{ll}\text { TLH } & \text { as } \\
\text { comparable }\end{array}$ \\
\hline 93 & VCA & 0,098 & 0,020 & $\begin{array}{ll}\text { SPC } \\
\text { comparable }\end{array}$ \\
\hline 94 & VDT & 0,493 & 0,242 & $\begin{array}{ll}\text { NSP } & \text { as } \\
\text { comparable }\end{array}$ \\
\hline 95 & VFG & 0,350 & 0,181 & \\
\hline 96 & VGS & 1,907 & 0,820 & \\
\hline 97 & VID & 0,757 & 0,272 & \\
\hline 98 & VIS & 1,289 & 0,500 & \\
\hline 99 & VKP & 0,877 & 0,131 & \\
\hline
\end{tabular}

All three above tables and data show that values of equity and asset beta in the three cases of changing competiotor size have certain fluctuation.

Comparing statistical results in 3 scenarios of changing leverage:

Table 5. Statistical results (FL in case 1) (source: VN stock exchange 2012)

\begin{tabular}{|l|l|l|l|}
\hline $\begin{array}{l}\text { Statistic } \\
\text { results }\end{array}$ & $\begin{array}{l}\text { Equity } \\
\text { beta }\end{array}$ & $\begin{array}{l}\text { Asset beta (assume debt beta }= \\
\text { o) }\end{array}$ & $\begin{array}{l}\text { Differenc } \\
\text { e }\end{array}$ \\
\hline MAX & 2,089 & 1,162 & 0,927 \\
\hline MIN & $-1,712$ & $-1,377$ & $-0,335$ \\
\hline MEAN & 0,747 & 0,371 & 0,375 \\
\hline VAR & 0,158 & 0,073 & 0,178 \\
\hline Note: Samle size 99 firms & \\
\hline
\end{tabular}

Table 6. Statistical results (FL in case 2) (source: VN stock exchange 2012)

\begin{tabular}{|l|l|l|l|}
\hline $\begin{array}{l}\text { Statistic } \\
\text { results }\end{array}$ & $\begin{array}{l}\text { Equity } \\
\text { beta }\end{array}$ & $\begin{array}{l}\text { Asset beta (assume debt beta }= \\
\text { o) }\end{array}$ & $\begin{array}{l}\text { Differenc } \\
\text { e }\end{array}$ \\
\hline
\end{tabular}


Huy. D. T. N. 2016. Selecting Various Industrial Competitors Affect The Risk Level of Viet Nam Manufacturing Material Industry During and After The Global Crisis 2007-2009

\begin{tabular}{|l|l|l|l|} 
MAX & 2,089 & 1,445 & 0,6434 \\
\hline MIN & $-1,712$ & $-1,377$ & $-0,3354$ \\
\hline MEAN & 0,786 & 0,392 & 0,3935 \\
\hline VAR & 0,2931 & 0,1348 & 0,1583 \\
\hline \multicolumn{2}{|l}{ Note: Sample size $: 99$} \\
\hline
\end{tabular}

Table 7. Statistical results (FL in case 3) (source: VN stock exchange 2012)

\begin{tabular}{|l|l|l|l|}
\hline $\begin{array}{l}\text { Statistic } \\
\text { results }\end{array}$ & $\begin{array}{l}\text { Equity } \\
\text { beta }\end{array}$ & $\begin{array}{l}\text { Asset beta (assume debt beta }= \\
\text { o) }\end{array}$ & $\begin{array}{l}\text { Differenc } \\
\text { e }\end{array}$ \\
\hline MAX & 2,089 & 1,162 & 0,9268 \\
\hline MIN & $-1,712$ & $-1,377$ & $-0,3354$ \\
\hline MEAN & 0,728 & 0,360 & 0,3678 \\
\hline VAR & 0,3605 & 0,1413 & 0,2192 \\
\hline \multicolumn{4}{|l}{ Note: Sample size $: 99$} \\
\hline
\end{tabular}

Based on the calculated results, we find out:

First of all, Equity beta mean values in all 3 scenarios are acceptable $(<0,8)$ and asset beta mean values are also small $(<0,4)$. In the case of reported leverage in 2011, equity beta max is 2,089 which is somewhat high in a few exceptional cases. If competitor size doubles, asset beta max decreases from 1,162 to 1,445 . Finally, when competitor size is slightly smaller, asset beta max keeps the same value of 1,162 .

The below chart 1 shows us : when competitive firm size decreases slightly, average equity beta value decrease slightly $(0,728)$ compared to that at the initial selected competitor $(0,747)$. Next, average asset beta decreases little (to 0,360 ). However, in case the competitor size doubles, the risk level of the selected firms is higher (0,392). Last but not least, the fluctuation of equity beta value $(0,293)$ in the case of doubling size competitors is smaller than ( $>$ ) the results in the rest 2 cases. And we could note that in the case competitor size slightly smaller, the risk is more dispersed $(0,361)$. 
Chart 1. Comparing statistical results of equity beta var and mean in three (3) scenarios of changing competitor size (source: VN stock exchange 2012)

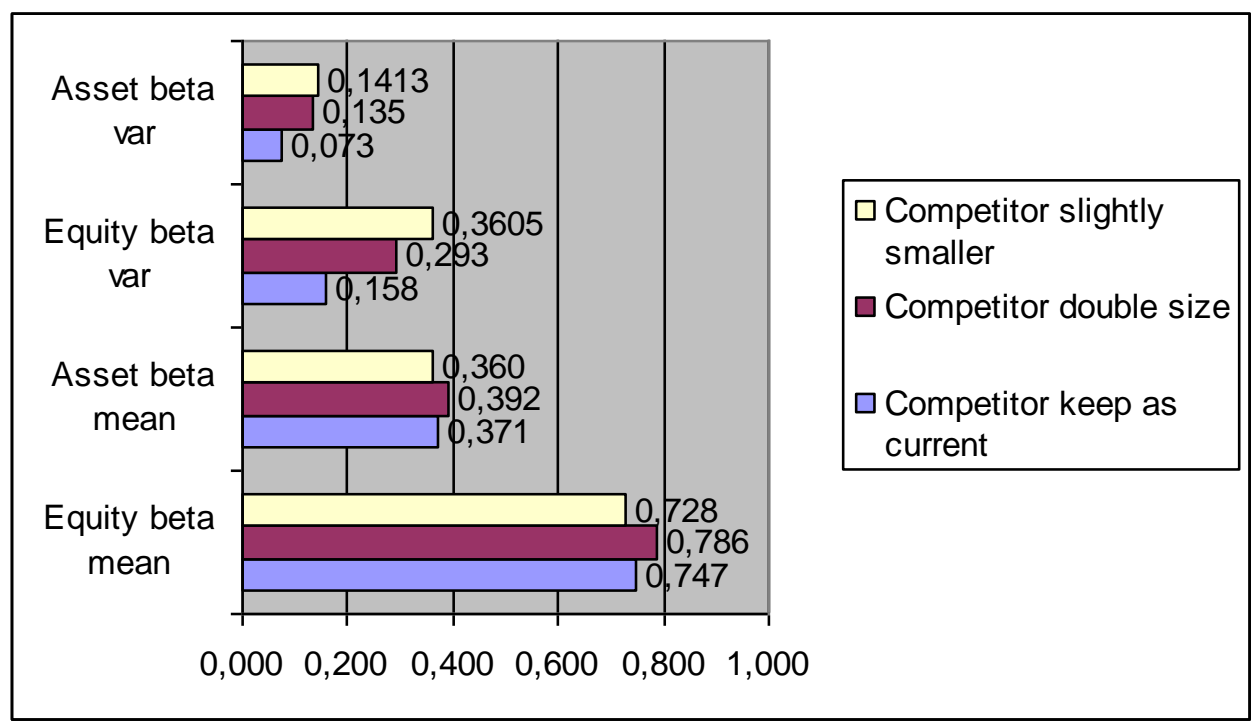

\section{Conclusion and Policy suggestion}

In conclusion, the government has to consider the impacts on the mobility of capital in the markets when it changes the macro policies and the legal system and regulation for developing the manufacturing material market. The Ministry of Finance continues to increase the effectiveness of fiscal policies and tax policies which are needed to combine with other macro policies at the same time. The State Bank of Viet Nam continues to increase the effectiveness of capital providing channels for manufacturing material companies as we could note that in this study when competitive firm size doubles, the risk level increases (equity beta mean value is estimated at: 0,786), and the 
equity beta var value $(0,293)$ is little lower than that in case competitor size as current $(0,303)$. Furthermore, the entire efforts among many different government bodies need to be coordinated. Finally, this paper suggests implications for further research and policy suggestion for the Viet Nam government and relevant organizations, economists and investors from current market conditions.

\section{Acknowledgements}

I would like to take this opportunity to express my warm thanks to Board of Editors and Colleagues at Citibank -HCMC, SCB and BIDV-HCMC, Dr. Chen and Dr. Yu Hai-Chin at Chung Yuan Christian University for class lectures, also Dr Chet Borucki, Dr Jay and my ex-Corporate Governance sensei, Dr. Shingo Takahashi at International University of Japan. My sincere thanks are for the editorial office, for their work during my research. Also, my warm thanks are for Dr. Ngo Huong, Dr. Ho Dieu, Dr. Ly H. Anh, Dr Nguyen V. Phuc and my lecturers at Banking University - HCMC, Viet Nam for their help. Lastly, thank you very much for my family, colleagues, and brother in assisting convenient conditions for my research paper.

\section{References}

1. Dexheimer, John., and Haugen, Carla, (2003), Sarbanes-Oxley: Its Impact on the Venture Capital Community, Minnesota Journal of Business Law and Entrepreneurship, Vol.2 No.1

2. Eugene, Fama F., and French, Kenneth R., (2004), The Capital Asset Pricing Model: Theory and Evidence, Journal of Economic Perspectives

3. Flifel, Kaouther., (2012), Financial Markets between Efficiency and Persistence : Empirical Evidence on Daily Data, Asian Journal of Finance and Accounting

4. Gao, Huasheng., Harford, Jarrad., and Li, Kai., (2013), Determinants of Corporate Cash Policy: Insights from Private Firms, Journal of Financial Economics

5. Huy, Dinh T.N., (2012), Estimating Beta of Viet Nam listed construction companies groups during the crisis, Journal of Integration and Development

6. Kale, Jayant R., Meneghetti, Costanza., and Sharur, Husayn., (2013), Contracting With NonFinancial Stakeholders and Corporate Capital Structure: The Case of Product Warantties, Journal of Financial and Quantitative Analysis

7. Litvak, Kate., (2008), Defensive Management: Does the Sarbanes-Oxley Act Discourage Corporate Risk-Taking?, Law and Economics Research Paper, No. 108

8. Ling, Amy., (2013), Tax Issues Relating to Intangibles, Asia-Pacific Tax Bulletin 
9. Lu, Wenling., and Whidbee, David A., (2013), Bank Structure and Failure,Journal of Financial Econoic Policy

10. Mukerjee, Kaushik., (2013), Customer-Oriented Organizations: A Framework for Innovation, Journal of Business Strategy

11. Shi, Mingtao., (2013), Capturing Strategic Competencies :Cloud Security as a Case Study, Journal of Business Strategy

12. Young, L., (2011), Market Orientation Processes, Australasian Marketing Journal Research

13. Ang, A., Chen, J., (2007), CAPM Over the Long Run: 1926-2001, Journal of Empirical Finance

14. Baker, Kent H., Singleton, Clay J., and Veit, Theodore E., (2011), Survey Research in Corporate Finance: Bridging The Gap Between Theory and Practice, Oxford University Press

15. ADB and Viet Nam Fact Sheet, 2010

\section{Other web sources}

1. http://www.mofa.gov.vn/vi/

2. http://www.hsx.vn/hsx/

3. www.tuoitre.com.vn;

4. www.saigontimes.com.vn;

5. www.mof.gov.vn

Exhibit 1. Inflation, GDP growth and macroeconomics factors (source: Viet Nam commercial banks and economic statistical bureau)

\begin{tabular}{llll} 
Year & Inflation & GDP & USD/VND rate \\
2011 & $18 \%$ & $5,89 \%$ & 20.670 \\
2010 & $\begin{array}{l}11,75 \% \text { (Es- } \\
\text { timated at Dec }\end{array}$ & $6,5 \%$ pected & 19.495 \\
& $2010)$ & & \\
2009 & $6,88 \%$ & $5,2 \%$ & 17.000 \\
2008 & $22 \%$ & $6,23 \%$ & 17.700 \\
2007 & $12,63 \%$ & $8,44 \%$ & 16.132 \\
2006 & $6,6 \%$ & $8,17 \%$ & \\
2005 & $8,4 \%$ & \multicolumn{3}{l}{} \\
Note & \multicolumn{3}{|l}{ approximately }
\end{tabular}

